\title{
LAS NEGOCIACIONES CON IRÁN SOBRE LA CUESTIÓN NUCLEAR
}

\author{
José Antonio Sainz de la Peña ${ }^{1}$ \\ UNISCI
}

\begin{abstract}
Resumen:
El artículo expone de forma resumida las conversaciones recientes entre Irán y la AIEA y el G5+1, mostrando las posiciones y las dificultades para alcanzar un acuerdo sobre el programa nuclear iraní. Las sanciones tienen, a su vez importantes efectos en la economía iraní, pero no está claro que Irán esté dispuesto a ceder en su programa de enriquecimiento de uranio, ni que se hayan adoptado por ambas partes o se puedan adoptar serias medidas de fomento de confianza, estando ya Irán bajo crecientes sanciones y no habiendo clarificado por completo su programa nuclear.
\end{abstract}

Palabras clave: Irán, IAEA, G5+1, Estados Unidos, Unión Europea, negociaciones nucleares, sanciones.

Title in English: "Iran and Negociations on Nuclear Issues”.

\section{Abstract:}

The article presents a summary of the recent talks between Iran and the IAEA and the G5+1, explaining the positions adopted by the parties and the difficulties in reaching an agreement on the Iranian nuclear program. The sanctions adopted have a major impact on the Iranian economy; however it is not clear that Iran is willing to reach a compromise on its uranium enrichment program. The precondition for a successful negotiation, an atmosphere of confidence and trust, has not been achieved. Iran is under increasing sanctions and Iran has not clarified its entire nuclear program.

Keywords: Iran, IAEA, G5+1, United States, European Union, Nuclear Negotiations, Sanctions.

Copyright (C) UNISCI, 2012.

Las opiniones expresadas en estos artículos son propias de sus autores, y no reflejan necesariamente la opinión de UNISCI. The views expressed in these articles are those of the authors, and do not necessarily reflect the views of UNISCI.

\footnotetext{
${ }^{1}$ José Antonio Sainz de la Peña es investigador senior de UNISCI.

E-mail: esedelape@hotmail.com.

http://dx.doi.org/10.5209/rev_UNIS.2012.n29.406"u
} 


\section{Las conversaciones de Irán con la AIEA y el G5+1}

Las conversaciones entre la Comunidad Internacional y la República Islámica de Irán (RII) sobre el programa nuclear iraní se han desarrollado en dos planos paralelos. En el primero, fundamentalmente técnico, el interlocutor de la RII ha sido la Agencia Internacional de la Energía Atómica (AIEA) y los encuentros han estado basados en los informes de los inspectores de la Agencia que regularmente visitan Irán. En el segundo, básicamente político, el interlocutor ha sido el llamado Grupo 5+1 (G 5+1), es decir, los cinco países miembros permanentes del Consejo de Seguridad (CS) de las Naciones Unidas más Alemania. El puente de enlace entre ambos planos ha sido, precisamente, el CS; la AIEA enviaba sus informes al CS, éste, basándose en los informes, votaba resoluciones y sanciones y el G5+1 las aplicaba.

Los resultados del trabajo del primer plano han sido los informes trimestrales de las inspecciones de la AIEA, cuya conclusión puede resumirse en "lo que las autoridades iraníes nos ha dejado ver, está bien; de lo que nos ha impedido ver, no podemos opinar, pero desconfiamos". En cuanto al segundo plano, excepto un principio de acuerdo obtenido en 2008, - que fracasó inmediatamente por la oposición de los máximos dirigentes de la RII-, las sucesivas reuniones se cuentan por desacuerdos y fracasos. El resultado se ha debido, por una parte, a la actitud inflexible de los negociadores iraníes en la defensa de "sus derechos inalienables al uso pacífico de la energía nuclear" y, por otra, a la desconfianza del G5+1 de que detrás del "uso pacífico" se oculte un programa de armamento nuclear.

Las conversaciones de diciembre de 2010, en Ginebra, y de enero de 2011, en Estambul, tuvieron un estrepitoso fracaso. Ambas partes mantuvieron con dureza sus respectivas posturas y los negociadores del G5+1 calificaron las condiciones previas de Irán de inaceptables. Las negociaciones no se reanudaron hasta quince meses después, en abril de 2012. ¿Qué había pasado durante esos quince meses y por qué se reanudaban las conversaciones? La respuesta es sencilla: los informes de la AIEA habían acusado a la RII de mentir, los Estados Unidos y la Unión Europea habían establecido contra Irán nuevas sanciones económicas y financieras, cada vez más estrictas, e Irán estaba empezando a sufrir las consecuencias.

\section{La AIEA e Irán}

La AIEA publicó, en mayo de 2011, un informe en el que decía que tenía pruebas de que la RII trabajaba en un iniciador para un explosivo nuclear. Además presentaba en él siete áreas de "preocupación" del programa nuclear por su posible aspecto militar, "no pacífico". La Agencia redactó un informe idéntico en noviembre en el que, además, confirmaba que Irán había conseguido progresar en su programa nuclear a pesar de los ataques cibernéticos contra sus instalaciones Entre los días 29 y 31 de diciembre y los 21 y 22 de febrero un equipo de la AIEA visitó Teherán. Para Irán las visitas fueron "constructivas y positivas"; para la Agencia fue un fracaso, ya que los dirigentes iraníes no contestaron a las preguntas sobre los temas pendientes, no fue autorizada para hablar con los científicos que trabajan en el programa nuclear ni a entrar en ciertas instalaciones, en particular en la base militar de Parchin, donde se sospecha que la RII ha llevado a cabo ensayos sobre el iniciador nuclear. El Ministerio de Asuntos Exteriores (MAE) de Irán invitó a la delegación de la AIEA a seguir más tiempo en Teherán, pero el equipo de la AIEA se negó. El único acuerdo alcanzado fue el de continuar con el diálogo. 
Un nuevo informe de la AIEA, en febrero, fue igual que los anteriores; la sola diferencia fue que se decía que la instalación subterránea de Fordo había aumentado su producción de uranio enriquecido al 20\%. La Agencia pidió que la RII no pusiese precondiciones para nuevas conversaciones y que facilitase a sus inspectores la entrada a Parchin. El 28 de febrero Soltanieh, el embajador representante del gobierno iraní ante la Agencia, insinúa que se podría incluir Parchin en una visita, Irán da a entender, el 7 de marzo, que autorizaría la visita a Parchin, pero el 1 de abril, Soltanieh se desdice afirmando que una cosa es la cooperación con la Agencia y otra que ésta pueda entrar en Parchin cuando quiera. El mismo día 7 de marzo, fotografías de satélite indican que hay actividad desacostumbrada en la instalación; al parecer se está limpiando la zona. El 9 de mayo, nuevas imágenes de satélite dan cuenta que la zona ya está limpia.

El 20 de abril la RII anuncia que está dispuesta para entablar negociaciones. Éstas se celebran en Viena, los días 14 y 15 del mismo mes. El negociador de la Agencia, Nackaerst, Director General del Departamento de Salvaguardias, dijo que el encuentro había ido bien y que él era optimista (véase más adelante la reacción iraní). Después de la reunión no hubo comentarios, pero se la calificó como "un intento para lograr un acuerdo sobre el procedimiento para resolver todos los temas pendientes" es decir, "las siete áreas de preocupación", y volver a pedir a Teherán que permitiese el acceso a personas, documentación e instalaciones. El Director, General de la AIEA, Yukita Amano, insistió en que la prioridad para la Agencia era el acceso a Parchin. No se firmó ningún acuerdo.

El 21 de mayo, Amano viajó a Teherán por primera vez, para entrevistarse con el MAE, Salehi, el principal negociador con el G 5+1, Yalili, y el jefe de la Organización Atómica iraní, Abbasi. La última visita del Director de AIEA, entonces Al Baradai, fue en 2009. Todo hacía suponer que la visita, que tenía lugar un día antes del negociaciones RII - G5+1, fuese de gran importancia. Sin embargo, Amano declaró que sólo se había logrado "un inicio de acuerdo estructurado" para permitir el desarrollo de las inspecciones, pero que no se había firmado nada., ya que subsistían "las diferencias". Abbasi prometió mayor colaboración con la Agencia, el día 17 Yalili había asegurado que Irán no cedería ni "una iota" de sus derechos y Salehi dijo que las entrevistas habían sido "un gesto de buena voluntad". Para los occidentales, la visita había sido un fracaso y las conversaciones seguían en punto muerto.

En un nuevo informe, publicado el 25 de mayo, la AIEA señalaba que sus inspectores habían encontrado muestras de uranio enriquecido al $27 \%$ en Fordo. Con gran prudencia, la Agencia suponía que ese grado de pureza, algo nuevo hasta el momento, podría deberse a un "error del operador", a algo no deseado ${ }^{2}$.

\section{La República Islámica frente a la AIEA}

La RII contestó a los sucesivos informes de la AIEA de la forma acostumbrada: eran "desequilibrados", tenían "motivación política" y estaban basados en "falsedades" y en documentos "fabricados", no verdaderos. Soltanieh, el 9 de noviembre de 2011, afirmaba que Amano, con esos informes, estaba jugando un juego peligroso y el 19 decía que Irán no retrasaría su programa nuclear ni un segundo. Los dirigentes iraníes continuaban diciendo que

\footnotetext{
${ }^{2}$ El trabajo y el tiempo necesario para enriquecer el uranio no es proporcional a la diferencia de niveles de enriquecimiento. Cuesta mucho más pasar del 3\% (nivel de combustible para producir electricidad) al 20\% (nivel para la producción de isótopos médicos o de uso industrial), que de este nivel al 90\% (nivel militar o del explosivo nuclear). Conseguido el enriquecimiento al $20 \%$ es posible llegar al $90 \%$ con rapidez.
} 
colaboraban completamente con la AIEA, pero no atendían sus recomendaciones, en especial la firma del Protocolo Adicional al Tratado de No Proliferación (TNP), que permitiría las inspecciones sin aviso previo.

El Director de la Organización Atómica, Abbasi, anunciaba el 2 de septiembre que la producción de uranio enriquecido al $20 \%$ sobrepasaba ya las necesidades iraníes para el reactor con finalidad médica de Teherán ( pero el 11 de abril se contradice asegurando que sólo se había enriquecido lo justo para el uso médico) y el 29 de febrero declaraba que Irán no podía autorizar la inspección de Parchin ya que esa base militar no es una instalación nuclear y, por lo tanto, la AIEA no tiene competencia sobre ella. El 9 de abril insistía en que en Parchin no se realizan actividades nucleares, al tiempo que revelaba que Irán había triplicado la capacidad de enriquecimiento de uranio, gracias a centrifugadoras de tercera generación.

El 28 de febrero Salehi ofrece a la AIEA una nueva visita a Teherán para tratar de implantar un nuevo mecanismo para solucionar las cuestiones pendientes. Merece la pena recordar que en febrero de 2008, como resultado de un "Plan de Trabajo" entre la AIEA y la RII para aclarar los asuntos pendientes, las autoridades iraníes declararon que ya todo estaba solucionado y no existían más temas pendientes; cuatro años más tarde el MAE iraní quería, por fin, resolverlos.

El 9 de abril, el Presidente Ahmadineyad explicó que Irán estaba preparado para nuevas conversaciones siempre que éstas se tuviesen dentro del marco del TNP y del estatuto de la AIEA; el 14 de abril las autoridades iraníes declaraban que estaban listas para revisar su decisión de enriquecer uranio al nivel el $20 \%$ si Occidente lo suministraba; el 20 de abril Teherán enviaba una carta a la AIEA diciendo que estaba dispuesto a iniciar nuevas negociaciones; y el 30 Soltanieh expresaba su deseo de que las conversaciones levantasen todas las "ambigüedades" y resolviesen todas las cuestiones pendientes. Amano aceptó la oferta. Sin embargo, el mismo Soltanieh decía, el 4 de mayo, que no había ninguna razón para cerrar Fordo, añadiendo que el enriquecimiento de uranio continuaría y, el día 12, que la suspensión de las actividades nucleares era imposible.

El 9 de mayo, después de la reunión de Viena, Soltanieh, alineándose con una declaración previa de Salehi, evaluaba el resultado de la conferencia diciendo que para Irán la prioridad era encontrar un marco para resolver los asuntos pendientes y aclarar ambigüedades. En una conferencia de prensa, junto con el negociador de la AIEA, Nackaerst, Soltanieh afirmaba que se habían realizado algunos progresos para establecer tal marco, dentro del que se podría incluir el asunto de la inspección de Parchin. Pero, a continuación, el 14, decía que Irán no puede aceptar condiciones que vayan más allá del TNP, con lo que anulaba la posibilidad de aceptar la puesta en marcha del Protocolo Adicional e, indirectamente, la inspección de Parchin.

Como contrapunto, las autoridades iraníes acusaron a la AIEA de filtrar informaciones que habían estado en el origen de los asesinatos de científicos que trabajaban en el programa nuclear.

\section{La República Islámica v el G 5+1}

Conocido el informe de noviembre de la AIEA, en el que la Agencia confirmaba e insistía en la falta de colaboración de Irán, Estados Unidos y sus aliados anunciaron, el día 21, que 
aumentaban las sanciones contra Teherán. Estas nuevas sanciones estaban dirigidas contra la industria del petróleo, se intentaba cortar el acceso de Irán al sistema financiero internacional y a dificultar la inversión extranjera en la industria petroquímica, el segundo pilar de la economía iraní. En el primer caso, el Banco Central de Irán, el Merkasi, a través del cual la RII realiza sus transacciones internacionales, todavía no era sancionado.

El 31 de diciembre, el presidente Obama firmó una ley intensificando las sanciones imponiendo sanciones a las instituciones financieras extranjeras que trabajasen con Irán; esas instituciones tenían que elegir entre hacer negocios con la RII o con los Estados Unidos. En la práctica, esta ley bloqueaba al Banco Merkazi y forzaba a los países importadores de petróleo iraní a buscar nuevas fuentes de suministro.

Por su parte. La Unión Europea, el día 23, prohibía comprar crudo y productos petroquímicos iraníes a partir del 1 de julio, así como exportar a ese país tecnología y maquinaria relativa a los sectores prohibidos, prohibía asegurar los petroleros que transportasen petróleo y congelaba los activos del Banco Merkazi. Los jefes de gobierno de Gran Bretaña, Alemania y Francia, en una declaración conjunta, declaraban que hasta que la RII no se sentase a negociar mantendrían las sanciones. El mismo día, los Estados Unidos incluían en las sanciones financieras al Banco Tejarat, la institución iraní número 23 en ser sancionada. El Ministerio de Exteriores iraní calificó la decisión de la UE de "ilegal e injustificable" y el Maylis, el día 26, amenazó con cortar la exportación de petróleo a Europa y consideró una acción inamistosa la de los países árabes que aceptasen cubrir la falta de crudo iraní; la TV el 15 de febrero anunciaba que se cortaba el suministro a seis países uropeo, algo que desmintió inmediatamente. Irán sólo exportaba a la UE el $18 \%$ de su petróleo.

El 6 de febrero, una Orden Ejecutiva de Obama congelaba en los Estados Unidos toda propiedad del gobierno iraní, del Banco Merkazi y de todas las instituciones financieras iraníes y avisaba a otras naciones sobre las sanciones que se les podría imponer si seguían comerciando con Irán.

El 15 del mismo mes, Catherine Ashton recibió una carta de Yalili en la que confirmó la aceptación por Irán de una oferta de la UE, hecha en octubre, para mantener conversaciones "constructivas" sobre el programa nuclear; el 6 de marzo, Ashton contestó a la carta positivamente, diciendo que la primera reunión debía centrarse en la construcción de "medidas de confianza"

El 17 de febrero los Estados Unidos y la UE expresan su confianza en que las conversaciones se reanudarían pronto, aunque todavía no estaba definida la estructura de las mismas, Ashton se declaraba "optimista pero cautelosa" y resaltaba la importancia de la "doble acción", es decir, negociar manteniendo las sanciones. Del lado iraní, Yilali manifestó que la RII estaba dispuesta al diálogo inmediato, pero Lariyani, el Presidente de la Asamblea Consultiva iraní, avisaba que las conversaciones serían inútiles si el G 5+1 ejercía presiones, señalando que la RII no haría concesiones.

El 8 de marzo el G 5+1 estuvo de acuerdo para volver a sentarse a una mesa para negociar con Irán, pero añadiendo que los inspectores de la AIEA debían ser autorizados a entrar en Parchin

El 14 de marzo Obama y el Primer Ministro británico Cameron pedían a la RII buena fé para negociar y el primero decía que la negociación era "la última ocasión" y que la "ventana 
de la diplomacia se estaba cerrando". Y, al día siguiente, la "Sociedad para las Telecomunicaciones Financieras Mundiales Interbancarias" (SWIFT en siglas anglesas), con sede en Bélgica, expulsa de la red a 30 bancos iraníes, incluidos el Banco Merkazi y el Melli o Nacional; esos bancos enviarían a través de SWIFT 2,3 millones de mensajes al año. La expulsión, que se realizaba por primera vez en la historia de SWIFT, afectaba profundamente a la economía iraní y aislaba todavía más al régimen. A finales de mes, Obama envío un mensaje al Ayatolá Jamenei, por medio del Primer Ministro turco Erdogan, en el que insistía en la urgencia de que Irán negociase seriamente.

En esta tesitura, los Estados Unidos y la UE establecieron el 7 de abril sus objetivos en las negociaciones: cerrar la instalación de Fordo y desmantelarla más tarde; parar el enriquecimiento de uranio al 20\%; enviar fuera de Irán el uranio al $20 \%$ almacenado. Indirectamente, se estaba legitimando el enriquecimiento al 3\%, pero siempre bajo la vigilancia de la AIEA y en el marco de las resoluciones de las UN; si se demostraba que el programa nuclear no tenía una parte militar, el enriquecimiento al $3 \%$ podría continuar.

El día 9, el Presidente iraní, Ahmadineyad, manifestó que la RII estaba dispuesta a mantener conversaciones sobre su programa nuclear "pacífico" con el G5+1, pero dentro de los límites del TNP y el día 11, Yalili, el secretario del Consejo Supremo de Seguridad Nacional iraní, máximo negociador ante el G5+1, anunciaba que haría nuevas propuestas y que propondría nuevas iniciativas en las conversaciones, pero no decía cuales. Además denunciaba que las sanciones contra Irán eran inútiles y que el lenguaje del reto y la presión no daría resultados.

Ambas partes esperaban que las negociaciones fuesen largas, paso a paso y con concesiones recíprocas, pero las posiciones eran muy distantes. La del G5+1 eran los objetivos fijados el día 7; las de la RII, la suspensión del embargo de petróleo y de las sanciones económicas $\mathrm{y}$, en contrapartida, sugería la interrupción temporal del enriquecimiento al 20\%. Sin embargo, la situación era muy diferente a la de las conversaciones anteriores: por un lado Irán tenía ahora una gran cantidad de uranio enriquecido almacenado, tanto al 3\% como al20\%, y en la instalación de Fordo había multiplicado su capacidad de producción; pero, por otra parte, las sanciones dañaban seriamente su economía.

Yalili, en otra carta a Ashton, le comunicaba que él era el representante personal del ayatolá Jamenei, lo que indicaba que esta vez, a diferencia del acuerdo de 2008, lo que Yalili firmase tendría el visto bueno del Guía Máximo de la RII.

La negociación tuvo lugar en Estambul, los días 13 y 14. Aunque tanto Ashton como Yalili se declararon satisfechos, la realidad es que no hubo resultados concretos. Para Ashton la reunión había sido "constructiva" y "útil”, pero hacía falta continuar con el diálogo, dando pasos concretos para restaurar la confianza. El TNP, que permite el uso pacífico de la energía nuclear, sería el marco correcto para desarrollar el proceso, pero el Tratado exige inspecciones. La frase de la negociadora de la UE sobre el uso pacífico de la energía nuclear fue utilizada posteriormente por Irán de forma sesgada afirmando que las potencias europeas reconocían el derecho iraní a la energía nuclear. Para Yalili, las conversaciones habían sido positivas, con un aumento del diálogo y la cooperación; el lenguaje de las amenazas ya no era válido. En consecuencia y teniendo en cuenta la cooperación de Irán con la AIEA, Yalili pidió a Ashton el levantamiento inmediato de todas las sanciones, cosa a la que Ashton se negó. 
Los únicos resultados tangibles de la reunión fueron el acuerdo de proseguir las negociaciones en Bagdad, el 23 de mayo, y que los segundos de ambos negociadores preparasen una agenda para ellas en Bagdad.

Durante la negociación, hubo un intento de conversaciones bilaterales Irán - Estados Unidos rechazadas por los iraníes, ya que, según Yalili, sólo el Guía Supremo puede tomar decisiones sobre las relaciones entre los dos países.

Dos días después, el 16, el MAE Salehi decía que Irán estaba dispuesto para resolver, en Bagdad, el problema nuclear "rápida y fácilmente" y sugería que Irán podría cambiar su política de enriquecimiento de uranio si los países occidentales levantaban las sanciones. Estambul, para Salehi, había sido un punto de inflexión en el diálogo y para Lariyani las conversaciones fueron un primer paso adelante. Además, Salehi, declaraba que Irán estaba "ávido" de encontrar un mecanismo que calmase las preocupaciones occidentales.

El mismo día 16, el jefe del Pasdaran decía que la frase de Ashton, antes citada, era una "carta ganadora" para Irán y, el día 18, el Maylis subrayaba el "reconocimiento de los derechos iraníes por los países occidentales en un documento oficial"; de las inspecciones como contrapartida, ni una palabra.

El día 24, los Estados Unidos implantaron una nueva sanción, esta vez contra las compañías que proporcionasen tecnología de comunicación a Irán. Por el contrario, el Fondo Monetario Internacional, el 2 de mayo, rehusó expulsarlo.

Por la parte del G5+1, el portavoz de política exterior de la UE, M.Mann, prometía que en las conversaciones de Bagdad no habría condiciones previas, mientras que,por Irán, Haddad-Adel, posible nuevo presidente del Maylis y consuegro del ayatolá Jamenei, declaraba que Irán esperaba que el G5+1 levantase las sanciones en esas conversaciones.

Basándose en diversas declaraciones iraníes sobre una actitud de mayor flexibilidad, los medios de comunicación occidentales publicaron que, después de Bagdad, la RII estaría dispuesta a firmar el Protocolo Adicional al TNP. El día 30 de abril, el presidente de la Comisión de Defensa y Política Exterior del Maylis, Aleidin Buruyerdi, desmintió inmediatamente las informaciones, diciendo que el Protocolo necesitaría la aprobación del Gobierno y del Parlamento y que éste no lo aprobaría ya que ni lo discutiría.

Al mes siguiente, el 4 de mayo, Soltanieh declaraba que no había razones para cerrar Fordo, añadiendo "una cosa está clara, el enriquecimiento nunca se suspenderá"; el 9, el ayatolá Amoli Lariyani aseguraba que la RII no abandonaría sus derechos nuclcleares por las presiones; el 11, el ayatolá Kashani insistía en que continuar con las sanciones demostraba la falta de sinceridad de los Estados Unidos y Ahmadineyad exigía que las potencias occidentales abandonasen el lenguaje de la fuerza; el 12, Yalili amenazaba con el fracaso de las negociaciones si Occidente pedía suspender el enriquecimiento. Al día siguiente precisaba que cualquier mal cálculo del G5+1 las llevaría al fracaso.

Como contrapunto, los Estados Unidos pidió a Irán que probase su sinceridad dando pasos "urgentes y prácticos" cumpliendo con sus obligaciones; la UE que suspendiese el enriquecimiento; y Ashton, dando pruebas de optimismo, esperaba, el 11 de mayo, resultados concretos y que Bagdad fuese el principio del fin de la disputa. El G5+1, a su vez, manifestaba, el 18 de mayo, que las conversaciones eran exploratorias, se quería comprobar las intenciones de Irán, no esperando grandes resultados de las mismas y, dado que 
consideraba que este país se sentaba a la mesa forzado por las sanciones, no estaba dispuesto a levantarlas ni a aplazarlas. Sin embargo, la UE sugería que se podría levantar la prohibición sobre los seguros de los petroleros que transportan crudo iraní, medida que estaba causando gran impacto sobre las exportaciones de Irán.

Yalili, el día 18, al exponer que la RII no cedería ni una pulgada en sus derechos, añadió que los que creen que se puede presionar a pueblo iraní e impedir su progreso por medio de sanciones están equivocados. Después de la reunión de Estambul, Yilali había afirmado que el punto crucial para toda negociación, incluida la de Bagdad, que influiría en las futuras negociaciones y cooperación, era el levantamiento de las sanciones. La RII sólo pedía los derechos reconocidos en el TNP.

Velayati, ex MAE y consejero del Guía, manifestaba, el día 19, que la RII había decidido que la conferencia de Bagdad se realizase en el marco de las regulaciones internacionales y del respeto a los derechos iraníes, la RII no toleraría presiones y que si el G5+1 no intentaba imponer sus "ilegales" puntos de vista, las conversaciones tendrían éxito. El 20, Buruyerdi pensaba que la reunión no sería definitiva, sino un inicio del fin del problema y exigía al G5+1 que cambiase la política de la confrontación por la de interacción; lo malo es que en su declaración llamaba "gobierno sionista" al de los Estados Unidos, lo que no auguraba un buen principio.

El mismo día, 203 diputados del Maylis aprobaban una resolución en la que pedían al G5+1 respeto para los derechos de Irán, acabar con la influencia de los sionistas, cambiar la política de confrontación por la de colaboración, que tomase medidas para ganar la confianza de Irán y que resistiese a las demandas irrazonables. En caso contrario, la política de presiones recibiría la respuesta adecuada.

Por el G5+1, su portavoz M. Mann manifestó que el éxito de las conversaciones dependía, exclusivamente, de Irán.

El 22, los medios iraníes hablaban de diferencias entre los miembros del G5+1. Al parecer existían dos paquetes de propuestas para las conversaciones. Por un lado, los países occidentales, que mantenían los objetivos del 7 de abril y ofrecían a Irán incentivos menores; por otro, la posición de Rusia que prefería un proceso paso a paso en el que la RII iría tomando medidas para crear confianza - como, por ejemplo, congelar el número de centrifugadoras y restringir su uso- mientras que el G5+1 no impondría nuevas sanciones e iría suavizando las ya existentes. Esta propuesta ya fue hecha en febrero y, más tarde, presentada en las conversaciones de Estambul, donde "despertó interés".

Las conversaciones tuvieron lugar, en Bagdad, los días 23 y 24 de mayo. En ellas se presentaron dos propuestas: la del G5+1 y la de la RII.

La primera, resumidamente, pedía: cerrar la instalación nuclear de Fordo o, al menos, abrirla a la inspección de la AIEA; suspender la producción de uranio enriquecido al 20\%; exportar a otro país ese uranio ya almacenado. Como contrapartida: proporcionar piezas de recambio para la aviación civil iraní, que carece de ellas; ayudas para la seguridad nuclear en las instalaciones civiles; enviar uranio al $20 \%$ preparado para uso médico; reconocer el derecho iraní a un programa pacífico de la energía nuclear, siempre que Irán cumpliese con sus obligaciones con la AIEA. 
La propuesta iraní, que no se presentó por escrito, abarcaba cinco puntos, no todos relativos al asunto nuclear: seguridad nuclear y cooperación; temas regionales; lucha contra el tráfico de drogas; lucha contra los piratas somalíes; el tema estrella y la "línea roja" de la RII: el derecho a enriquecer uranio sin limitaciones.

Para Irán la propuesta del G5+1 era "desequilibrada"; para el G5+1 la de Irán insuficiente.

Además de ambas propuestas, se debatieron otros asuntos hasta el último minuto. Irán sugirió que podría parar, temporalmente, la producción de uranio al $20 \%$ y pidió el levantamiento de todas las sanciones. El G5+1 se negó a levantarlas y, también, a posponer las previstas para el 1 de julio.

El único acuerdo, alcanzado "in extremis", fue volver a reunirse en Moscú el 18 y 19 de junio. Sin embargo, los asistentes a las conversaciones hablaron de haber llegado a un "terreno común y de "atmósfera constructiva". Mann dijo que el asunto era tan importante que no se podía arreglar en "una noche". Ashton habló del terreno común y de la decisión de seguir adelante, a pesar de las diferencias, y Yalili calificó las conversaciones de positivas, pero señalando que el principal obstáculo se había centrado en que los occidentales no aceptaban el derecho iraní a enriquecer uranio; si lo hubiesen reconocido habría colaboración.

\section{A modo de conclusión}

En las recientes conversaciones sobre el programa nuclear de Irán, los dirigentes iraníes han introducido un curioso argumento: la utilización de un aspecto "espiritual" frente al materialismo occidental: la teoría del armamento nuclear como pecado.

Esa teoría empezó, en 2005, con un edicto religioso - "fatwa"- del ayatolá Jamenei en el que se declaraba que la "fabricación, el almacenamiento y el empleo del arma nuclear" es "haram", es decir, prohibido por el Islam ${ }^{3}$. En 2012, el argumento ha sido empleado de nuevo en numerosas ocasiones. Tanto el propio Jamenei, en su mensaje del Año nuevo persa, como Salahi, en la Conferencia de Desarme de Ginebra o en un artículo publicado en el Washington Post, como Yalili en las conversaciones de Bagdad, como diversos parlamentarios del Maylis o algunos intelectuales extranjeros musulmanes han afirmado que el arma nuclear es un gran pecado, que es peligrosa e inútil, que no tiene cabida en la doctrina militar iraní y que, aunque Irán posee la tecnología y los medios para tenerla, ni la tiene ni la tendrá nunca. Finalmente, como el Guía ha dicho que el arma nuclear es "haram", en Irán no hay armas nucleares y, por lo tanto, no son necesarias las inspecciones de la AIEA.

Con respecto a las sanciones, los iraníes las califican de fracaso, dicen que, incluso, han sido beneficiosas pues han obligado a realizar tareas que antes no hacían o hacían mínimamente, como la producción de gasolinas, de las que Irán ha pasado de ser importador a exportador. Ahmadineyad se expresó de esa manera el 3 y el 10 de abril, añadiendo que Irán tiene tanto dinero en divisas por las exportaciones de años pasados que puede permitirse estar años sin exportar crudo y el Ministro de Petróleo aseguró que a Irán no le faltan compradores. La realidad es que, según la OPEP, Irán en los tres primeros meses había reducido sus exportaciones en un $12 \%$, que los países asiáticos reducían sus compras en un tercio, que a

\footnotetext{
${ }^{3}$ Conviene subrayar que Pakistán, Estado nuclear, es también un República Islámica.
} 
consecuencia del embargo financiero Irán tenía grandes problemas para vender y para comprar en el extranjero (compra fuera unos 62.000 millones de dólares y vende sólo 44.000), que la India (13\% del petróleo iraní) ha reducido sus compras en un $15 \%$ y que el $45 \%$ lo paga en rupias, no en dólares; como la rupia no es convertible, Irán tiene que usar ese dinero en adquirir productos indios, y que China ( $22 \%$ de las ventas) discute los medios de pago.

Como datos finales, cabe señalar que el presupuesto para este año, que empezó el 21 de marzo, es de 416.000 millones de dólares, con una variación del 0,4\% sobre el año anterior, pero que el presupuesto de Defensa ha aumentado el $127 \%$, que en la preparación del citado presupuesto se partía de 1 dólar por 11.000 riales, cuando ahora el cambio es de 1 por 17.000, aproximadamente, y que la inflación, que para Ahmadineyad es del 6\%, para las autoridades monetarias iraníes es de $21 \%$ (cifra que puede ser menos de la mitad de la inflación real) y sube a razón de $0,5 \%$ al mes, mientras que han ido bajando los subsidios del Estado. La situación económica de la RII es, simplemente, desastrosa y lo será aún más si entran en acción las sanciones previstas para julio.

La respuesta iraní a las sanciones ha sido, frente al exterior, responsabilizar a los países occidentales de los resultados de las mismas sobre la economía mundial y, en el interior, apelar a los dos pilares ideológicos del régimen: nacionalismo exaltado e islamismo chií con vocación de martirio.

Además, los dirigentes de la RII han amenazado, numerosas veces, con que, en el caso de ser agredidos tomarían represalias. Estas podrían ser atacar con sus misiles a Israel y a la treintena de bases militares que hay en la región, acciones contra los intereses americanos e israelíes en todo el mundo y el cierre del estrecho de Ormuz. Sin embargo, como ha dicho algún experto, "Irán tiene una gran boca, pero un pequeño garrote". Sus amenazas suenan a bravatas para el consumo interior e Irán seguirá con su política de siempre, de ir avanzando paso a paso, comprando tiempo con negociaciones, cediendo cuando no pueda más, pero teniendo a la vista sus objetivos: supervivencia del régimen, integridad del territorio y liderazgo regional.

Probablemente, las conversaciones seguirán, con altos y bajos, hasta el momento en el que la RII no tenga el arma nuclear, pero que esté en condiciones de tenerla en poco tiempo en caso necesario (el llamado "modelo Japonés") y cuando sus instalaciones nucleares, por sus medios de protección activos y pasivos, hayan alcanzado la "zona de impunidad" que les haga casi indestructibles.

Lo que es seguro es que, mientras haya negociaciones, no habrá guerra. 